Article

\title{
Strong and Thermostable Boron-Containing Phenolic Resin-Derived Carbon Modified Three-Dimensional Needled Carbon Fiber Reinforced Silicon Oxycarbide Composites with Tunable High-Performance Microwave Absorption Properties
}

\author{
Yu Sun * and Yuguo Sun * \\ Center for Composite Materials, Harbin Institute of Technology, Harbin, 150080, China \\ * Correspondence: sunyuhit88@163.com (Y.S.); sunyg@hit.edu.cn (Y.S.); Tel./Fax: +86-451-86402386 (Yuguo Sun)
}

Received: 6 February 2020; Accepted: 6 March 2020; Published: 11 March 2020

\begin{abstract}
This paper focuses on the preparation of boron-containing phenolic resin (BPR)-derived carbon modified three-dimensional (3D) needled carbon fiber reinforced silicon oxycarbide (SiOC) composites through a simple precursor infiltration and pyrolysis process (PIP), and the influence of PIP cycle numbers on the microstructure, mechanical, high-temperature oxidation resistance. The electromagnetic wave (EMW) absorption properties of the composites were investigated for the first time. The pyrolysis temperature played an important role in the structural evolution of the SiOC precursor, as temperatures above $1400{ }^{\circ} \mathrm{C}$ would cause phase separation of the SiOC and the formation of silicon carbide $(\mathrm{SiC})$, silica $\left(\mathrm{SiO}_{2}\right)$, and carbon. The density and compressive strength of the composites increased as the PIP cycle number increased: the value for the sample with 3 PIP cycles was $0.77 \mathrm{~g} / \mathrm{cm}^{3}, 7.18 \pm 1.92 \mathrm{MPa}$ in $\mathrm{XY}$ direction and $9.01 \pm 1.25 \mathrm{MPa}$ in $\mathrm{Z}$ direction, respectively. This composite presented excellent high-temperature oxidation resistance and thermal stability properties with weight retention above $95 \%$ up to $1000^{\circ} \mathrm{C}$ both under air and Ar atmosphere. The minimal reflection loss (RLmin) value and the widest effective absorption bandwidth (EAB) value of as-prepared composites was $-24.31 \mathrm{~dB}$ and $4.9 \mathrm{GHz}$ under the optimization condition for the sample with 3 PIP cycles. The above results indicate that our BPR-derived carbon modified 3D needled carbon fiber reinforced SiOC composites could be considered as a promising material for practical applications.
\end{abstract}

Keywords: SiOC; pyrolysis temperature; PIP cycle; microwave absorption

\section{Introduction}

With the rapid development of technology, low-cost, lightweight, and reusable thermal protection materials for aerospace applications are in high demand to protect vehicles from damage under extreme conditions [1]. According to previous literature, carbon bonded carbon fiber composites (CBCF) have attracted much attention due to their low density and low thermal conductivity, and are considered a promising candidate for thermal insulation. $\mathrm{CBCF}$ is commonly prepared by vacuum or pressure filtration followed the pyrolysis method using chopped carbon fibers and phenolic resin (PR) as raw materials [2,3]. Although numerous efforts have been made to research $\mathrm{CBCF}$ composites, the intrinsic problems of $\mathrm{CBCF}$ composites severely restrict them for practical application, namely weak mechanical and poor oxidation resistance under high-temperature conditions [4].

From the perspective of the mechanical property, the three-dimensional (3D) needled carbon fiber preform shows promise for overcoming the poor mechanical property of pristine chopped 
carbon fibers [4,5]. In addition, the introduction of ceramic has been confirmed as an effective way to improve the high-temperature oxidation resistance of the composites up to now [6,7]. Notably, the pristine 3D needled carbon fiber preform is too flexible to operate, and thus direct incorporation of the ceramic phase would result in the structural distortion of the pristine preform. In order to improve the operability of the pristine preform, incorporation of resin-derived carbon through a simple vacuum infiltration and pyrolysis process is an effective method similar to the preparation of CBCFs composites. In addition, compared with common phenolic resin (PR), boron-containing phenolic resin (BPR) presents better thermal stability at high-temperature due to much higher B-O bond energy than that of the $\mathrm{C}-\mathrm{O}$ bond. Thus, it is reasonable to believe that introducing BPR-derived carbon into the pristine 3D needled carbon fiber preform through a simple method would enhance the high-temperature thermal stability of the composites compared to that of common PR pyrolyzed carbon [8]. From the ceramic point of view, polymer-derived ceramics (PDCs) have gained much attention, which could be attributed to their remarkable properties over conventional ceramics, such as low processing temperature and excellent properties, especially in thermal stability, high-temperature mechanics, and oxidation resistance $[9,10]$. Among all PDCs, apart from the amorphous phase with low thermal conductivity, the low-cost silicon oxycarbide (SiOC) ceramics possess inherent chemical durability, tunable mechanical and unusual high-temperature properties, which should be ascribed to its special structure, namely an anionic silica network, in which, two divalent $\mathrm{O}$ atoms are partially replaced by one tetravalent $C$ atom [11-13]. Hence, SiOC modified CBCF composites have been constructed and investigated by researchers [14], while there is no report on BPR-derived carbon modified 3D needled carbon fiber reinforced SiOC composites, which according to previous research have superior mechanical and high-temperature properties and could be used in high-temperature applications such as thermal protection systems.

Moreover, the functional performance of PDCs after high-temperature annealing is also desired in addition to the structural properties, and the electromagnetic wave (EMW) absorption properties of PDCs have aroused lots of attention in recent years [15-23]. For example, Hong et al. [21] successfully prepared porous PDC-SiC/ $\mathrm{Si}_{3} \mathrm{~N}_{4}$ composites by the unidirectional freeze casting of $\mathrm{Si}_{3} \mathrm{~N}_{4} /$ camphene slurries with various polycarbosilane (PCS) contents. The results showed that the EMW absorption properties of the composites could be effectively controlled by the PCS content, whose minimum reflection loss (RLmin) was as low as $-56.20 \mathrm{~dB}$ and the widest effective absorption bandwidth (EAB) was up to $7.00 \mathrm{GHz}$ for the sample with $20 \mathrm{wt}$.\% PCS. Yin and co-workers [22] fabricated nano $\mathrm{SiC}$-modified SiOC ceramics through pyrolysis of a mixture of liquid polysiloxane and nano $\mathrm{SiC}$ with an average grain diameter of $30 \mathrm{~nm}$, and the RLmin and widest EAB values were $-61.00 \mathrm{~dB}$ and $3.50 \mathrm{GHz}$ for the sample after it was annealed at $1400^{\circ} \mathrm{C}$, respectively. High-performance silicon boron carbonitride $(\mathrm{SiBCN})$ ceramics with excellent EMW absorption efficiency were obtained by Kong et al. [23] by pyrolysis of carbon-rich hyperbranched polyborosilazane precursors with pendant phenyl groups. The ceramics obtained at $1320^{\circ} \mathrm{C}$ showed the optimized EMW absorption performance with an RLmin value of $-71.80 \mathrm{~dB}$ and an EAB of $3.65 \mathrm{GHz}$. Therefore, it is expected that the BPR-derived carbon modified 3D needled carbon fiber reinforced SiOC composites might also attain good EMW absorbing properties because of the presence of carbon fibers with good electrical conductivity.

Based on these above points, the objective of the present work is therefore to investigate the microstructure and properties of BPR-derived carbon modified 3D needled carbon fiber reinforced SiOC composites through a simple precursor impregnation and pyrolysis (PIP) method for the first time. The influence of annealing temperature on the formation of the composition and microstructure of the products were analyzed. In addition, the effects of PIP cycle numbers on the morphology, microstructure, thermal stability, and mechanical and microwave absorption properties of BPR-derived carbon modified 3D needled carbon fiber reinforced SiOC composites were also investigated in detail. 


\section{Experimental Section}

\subsection{Preparation of BPR-Derived Carbon Modified 3D Needled Carbon Fiber Reinforced SiOC Composites}

First, the cubic samples (size: $10 \times 10 \times 10 \mathrm{~mm}^{3}$ ) used as substrate were cut from bulk anisotropic BPR-derived carbon modified 3D needled carbon fiber composites with densities of $0.28 \pm 0.05 \mathrm{~g} / \mathrm{cm}^{-3}$ and porosities of about $84 \%$. The above low-density C/C specimens were ultrasonically cleaned with ethanol for $10 \mathrm{~min}$ and dried at $80^{\circ} \mathrm{C}$ for $24 \mathrm{~h}$, and the preparation of SiOC precursor was through a co-hydrolysis method according to previous literature [4]. The detail for preparation of SiOC precursor was listed as following: $500 \mathrm{~g}$ methyltrimethoxysilane (MTMS, supplied by Zhengzhou ALFA Chemical Co., Ltd., Zhengzhou, China; 97\% purity) and $62.5 \mathrm{~g}$ dimethyldimethoxysilane (DMDMS, supplied by Zhengzhou ALFA Chemical Co., Ltd., Zhengzhou, China; 99\% purity) were dissolved in the solvent of $67.5 \mathrm{~g}$ ethyl alcohol (EtOH, purchased from Harbin Kecheng Chem. Co., Harbin, China; $99.9 \%$ purity) and $112.5 \mathrm{~g}$ distilled water (purchased from Harbin Kecheng Chem. Co., Harbin, China), and then $1.34 \mathrm{~g} \mathrm{HNO}_{3}(0.1 \mathrm{M}$, purchased from Harbin Kecheng Chem. Co., Harbin, China) was added to catalyze the gelation process, and then the above mixture was stirring for $20 \mathrm{~min}$ to form the SiOC precursor. Then the dried low-density C/C specimens were impregnated with the excess SiOC precursor for $60 \mathrm{~min}$ at room temperature using a vacuum infiltration device, and then the specimens filled with SiOC precursor were heated to $70^{\circ} \mathrm{C}$ for $48 \mathrm{~h}$ for cross-linking and curing. Then the cured specimens were pyrolyzed at high-temperature $\left(1200,1400\right.$, and $\left.1600^{\circ} \mathrm{C}\right)$ at a heating/cooling rate of $3^{\circ} \mathrm{C} / \mathrm{min}$ with a holding time of $60 \mathrm{~min}$ at the maximum temperature under a flowing Ar atmosphere. Finally, the BPR-derived carbon modified 3D needled carbon fiber reinforced SiOC samples were achieved after cooling to room temperature. In order to investigate the effect of PIP cycle numbers on the microstructure and properties of BPR-derived carbon modified 3D needled carbon fiber reinforced SiOC composites, the process of impregnating the BPR-derived carbon modified 3D needled carbon fiber specimens with the sol gelling, drying, and heating, was repeated 1,2, and 3 times, which were denoted as S1, S2, and S3, respectively.

\subsection{Characterization and Measurement}

The phase composition of as-prepared composites was analyzed by an X-ray diffractometer (XRD, PANalytical, Almelo, Holland). The carbon states in the samples were investigated from the Raman spectra through a JY HR-800 Raman microscope (Horiba Jobin Yvon, Paris, France) by irradiation with a $532 \mathrm{~nm}$ laser. The morphologies and microstructures of the composites were observed by the field-emission scanning electron microscopy (SEM, HELIOS NanoLab 600i, FEI Company, Hillsboro, OR, USA), transmission electron microscopy, and high-resolution transmission electron microscopy (TEM and HRTEM, Tecnai G2-F30, FEI Company, Hillsboro, OR, USA). The densities of samples with different PIP cycle numbers were measured by the Archimedes method using a high-accuracy electronic balance, and the details for the measurement were reported elsewhere [4,5]. The compressive behavior and strength were evaluated using an Instron 5569 universal test machine to acquire the mechanical properties of as-prepared composites, and the tested specimens with the dimension of $10 \times 10 \times 12 \mathrm{~mm}^{3}$ were uploaded at a constant rate of $0.5 \mathrm{~mm} / \mathrm{min}$. Notably, the average value was used to improve the accuracy and reliability of the data, which came from at least five samples for each kind of sample obtained in the same condition. The thermal stabilities of as-obtained composites both in air and Ar atmospheres were analyzed by the thermogravimetric analysis (TGA, SDT Q600, TA Instruments, Wilmington, DE, USA) with a heating rate of $5^{\circ} \mathrm{C} / \mathrm{min}$ up to the temperature of $1000^{\circ} \mathrm{C}$. To investigate the functional performance, namely the EMW absorption property of the composites, the electromagnetic parameters of samples with different PIP cycle numbers were measured at $2-18 \mathrm{GHz}$ using a vector network analyzer (Agilent N5230A, Agilent, Palo Alto, CA, USA) in a wax matrix with $50 \%$ weight loading. The samples were obtained from the composites homogeneously mixed with wax at $85{ }^{\circ} \mathrm{C}$ and then the homogeneous mixture was pressed into a toroidal mold with an inner diameter of $3.00 \mathrm{~mm}$ and an outer diameter of $7.00 \mathrm{~mm}$. After they were taken out of the mold, 
the measured samples were obtained, and the reflection loss (RL) was obtained using the relative complex permittivity $\left(\varepsilon_{\mathrm{r}}=\varepsilon^{\prime}-j \varepsilon^{\prime \prime}\right)$ and complex permeability $\left(\mu_{r}=\mu^{\prime}-j \mu^{\prime \prime}\right)$ by our self-programmed software, following equations according to the line theory [24-26]:

$$
\begin{gathered}
R L(d B)=20 \lg \left|\frac{Z_{\text {in }}-1}{Z_{\text {in }}+1}\right| \\
Z_{\text {in }}=\sqrt{\frac{\mu_{r}}{\varepsilon_{r}}} \tanh \left[j \frac{2 \pi f d}{c} \sqrt{\mu_{r} \varepsilon_{r}}\right]
\end{gathered}
$$

In which, $Z_{\text {in }}$ refers to the normalized input impendence of the absorber, $d$ stands for the thickness of the tested sample, $c$ represents the light velocity in free space, and $f$ is the frequency of the microwave frequency, respectively.

\section{Results and Discussion}

\subsection{Effect of Pyrolysis Temperature on the Composition and Microstructure of the Composites}

According to previous literature, the composition and microstructure of composites with the addition of SiOC precursor were strongly dependent on the pyrolysis temperature. Therefore, the effect of the pyrolysis temperature on the composition and microstructure of the as-obtained composites was investigated first $[11,13,27]$. Figure 1 shows the XRD patterns collected on the composites pyrolyzed at 1200,1400 , and $1600{ }^{\circ} \mathrm{C}$ for $60 \mathrm{~min}$. At low pyrolysis temperature $1200^{\circ} \mathrm{C}$, apart from the sharp diffraction peak at about $15^{\circ}$ from carbon, the term pattern of the products presents a completely amorphous structure only with the presence of amorphous silica centered at about $2 \theta=25^{\circ}$. After the pyrolysis temperature increased to $1400^{\circ} \mathrm{C}$, the (111) diffraction peak of cubic $\mathrm{SiC}$ at about $35^{\circ}$ starts to appear, and this diffraction peak becomes more evident when the pyrolysis temperature further increased to $1600{ }^{\circ} \mathrm{C}$, and other characteristic peaks of $\mathrm{SiC}$ could also be observed, including at about $42^{\circ}, 60^{\circ}$, and $72^{\circ}$, which could be attributed to the (200), (220), and (311) planes of standard cubic SiC (JCPDS No. 29-1129), respectively [28-30]. The above results confirm that the crystallization of $\beta$-SiC would be promoted by the increasing of pyrolysis temperature, and the pyrolysis temperature plays an important role in the composition of the composites after the introduction of SiOC. During the high-temperature treatment, the following reactions would occur within the structural evolution of the SiOC preceramic based on previous results $[11,13,27]$ :

$$
\begin{gathered}
\mathrm{SiOxCy} \rightarrow \mathrm{SiO}_{2} \text { (amorphous) }+\mathrm{SiC}+\mathrm{C}_{\mathrm{free}} \\
\mathrm{SiO}_{2} \text { (amorphous) }+3 \mathrm{C}_{\mathrm{free}} \rightarrow \mathrm{SiC}+2 \mathrm{CO}
\end{gathered}
$$

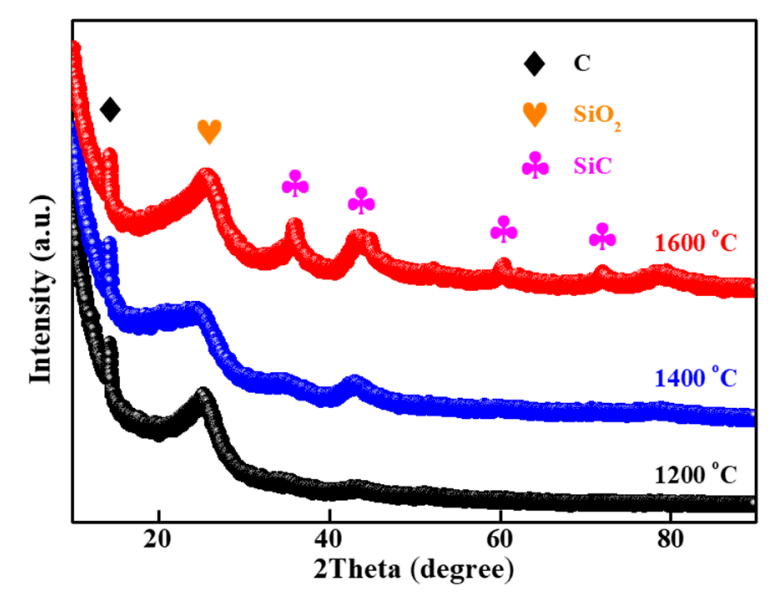

Figure 1. The XRD patterns of as-prepared composites after different pyrolysis temperatures. 
From the results shown, it can be seen that Reaction (3) occurs below $1400{ }^{\circ} \mathrm{C}$, if the temperature exceeds $1400^{\circ} \mathrm{C}$, Reaction (4) takes place.

Raman spectroscopy was carried out to evaluate the structural evolution of the free carbon phase within the as-prepared composites under different pyrolysis temperatures, and the results are shown in Figure 2. From the results, two obvious peaks could be observed, approximately 1350 and $1600 \mathrm{~cm}^{-1}$, referring to the typical D and G band corresponding to the disordered and graphitized carbon state, respectively [31]. The relative parameters for the above two bands are listed in Table S1, and the intensity ratio of the $D$ band to the $G$ band $\left(I_{D} / I_{G}\right)$ could be employed to evaluate the relative content of free carbon within the sample, and the higher value implies the higher relative content of free carbon compared with that of the graphitized carbon [32,33]. From the results listed in Table S1, the value of $I_{D} / I_{G}$ increases with the increase of pyrolysis temperature, suggesting that the relative content of free carbon increases as the pyrolysis temperature is increased. The value of $I_{D} / I_{G}$ could also be used to analyze the content of defects in the sample. The higher value indicates the larger content of defects in the sample, and thus it is reasonable to believe that the number of defects in the composites increases with pyrolysis temperature, which would be beneficial to improve the EMW absorption properties of the composites. In addition, the band around $2700 \mathrm{~cm}^{-1}$ could be assigned to two-phonon (2D) band, which refers to the graphitic ordering, which appears at $1400^{\circ} \mathrm{C}$ and becomes more evident at $1600^{\circ} \mathrm{C}$, demonstrating that the composites after above $1400^{\circ} \mathrm{C}$ heat-treatment would own relatively high graphitization degree, which would result in enhanced EMW absorption performance [27].

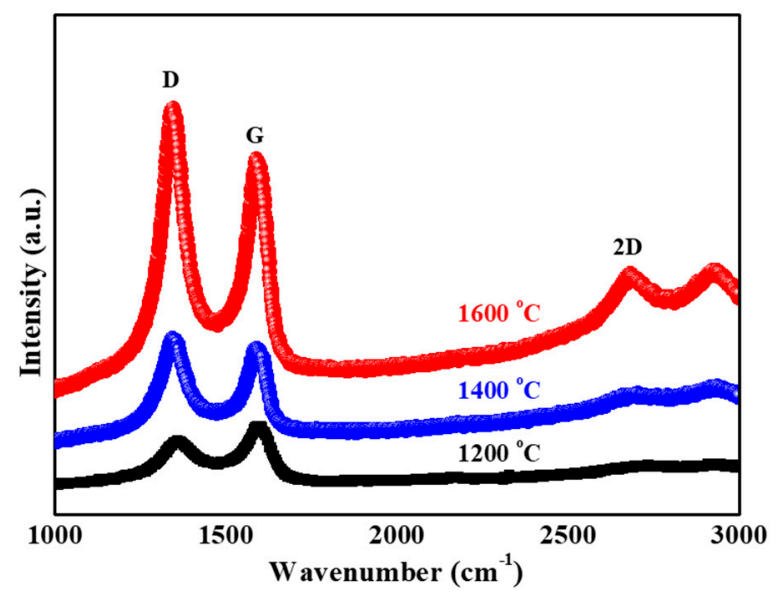

Figure 2. The Raman spectra of as-prepared composites after different pyrolysis temperatures.

To further analyze the microstructure of as-prepared composites, the TEM, HRTEM, and selected area electron diffraction (SAED) images are displayed in Figure 3. The low-resolution TEM image of the prepared composites is shown in Figure $3 \mathrm{a}$, the bulk SiOC ceramic and carbon fiber are marked. A closer look at the area marked A in Figure 3a is shown in the HRTEM image in Figure 3b. The presence of the lattice plane and the disordered zone is clearly visible, the distance between the planes of the selected area is about $0.25 \mathrm{~nm}$ in accordance with the (111) plane space of $\beta$-SiC, which is in agreement with the result of the XRD analysis [34,35]. Figure 3c depicts the SAED image of the disordered zone shown in Figure $3 b$, indicating an amorphous structure of carbon within the sample. In addition, the SAED image in Figure $3 \mathrm{~d}$ of the $\mathrm{SiC}$ phase displayed in Figure $3 \mathrm{~b}$ illustrates a single-crystal electron diffraction spot corresponding to $\beta$-SiC, indicating that $\mathrm{SiC}$ are formed within SiOC ceramic during the pyrolysis process, which is in accordance with the analysis of the XRD result and previous literature $[11,13,27]$. From the results, it can be concluded that the $\mathrm{SiOC}$ will decompose into $\mathrm{SiO}_{2}, \mathrm{SiC}$, and free carbon if the pyrolysis temperature is above $1400^{\circ} \mathrm{C}$. Therefore, the desired temperature for the preparation of BPR-derived carbon modified 3D needled carbon fiber reinforced SiOC composites is selected at $1400{ }^{\circ} \mathrm{C}$, which is favorable for maintaining the relatively low thermal conductivity of composites intended for use as thermal protection materials in harsh environments. 

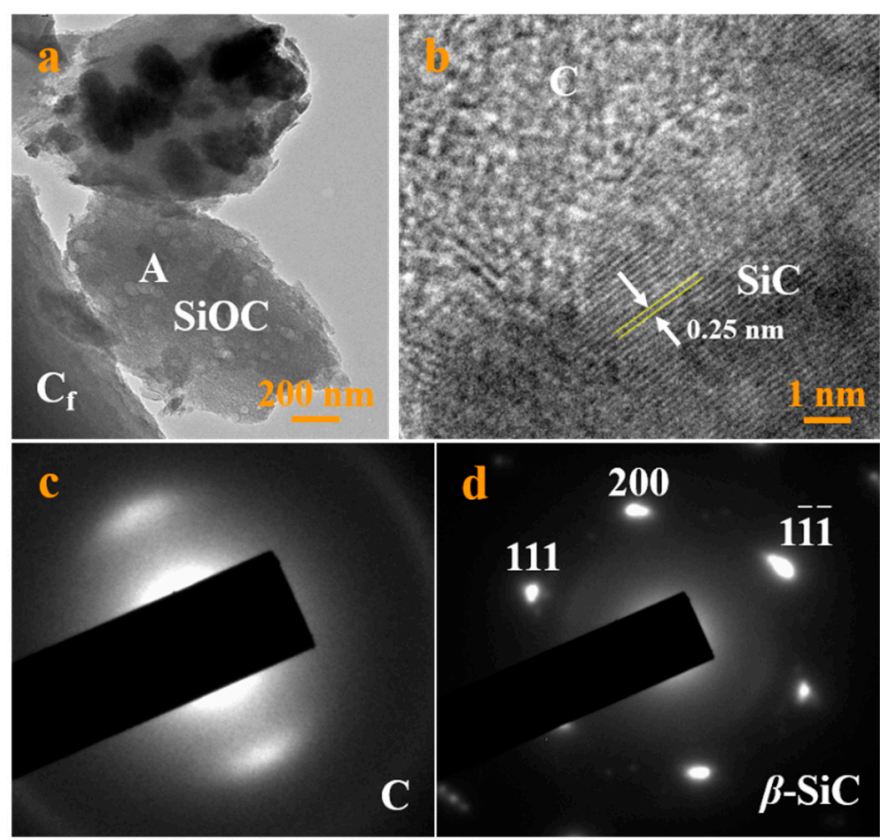

Figure 3. (a) TEM image of as-prepared composites obtained at $1600{ }^{\circ} \mathrm{C}$. (b) HRTEM image of SiOC within as-prepared composites obtained at $1600^{\circ} \mathrm{C}$. SAED images of (c) free carbon and (d) SiC within as-prepared composites obtained at $1600^{\circ} \mathrm{C}$.

\subsection{Influence of PIP Cycle Numbers on the Microstructure and Properties of the Composites}

According to the above results, the BPR-derived carbon modified 3D needled carbon fiber reinforced SiOC composites were achieved after annealing at $1400^{\circ} \mathrm{C}$, and three PIP cycle numbers $(1$, 2 , and 3 times) were selected to investigate the effect of PIP cycle numbers on the microstructure and properties of BPR-derived carbon modified 3D needled carbon fiber reinforced SiOC composites, and the three samples were marked as S1, S2, and S3, respectively.

Firstly, the influence of PIP cycle numbers on the densities of the as-prepared composites was evaluated, and the dependence of density on the numbers of the PIP cycle, as depicted in Figure 4. The pristine low-density BPR-derived carbon modified 3D needled carbon fiber composites was about $0.28 \mathrm{~g} / \mathrm{cm}^{3}$, and it can be clearly seen that the density of as-prepared composites increases with increasing PIP cycle number; this is in line with previous literature [4,5]. Concretely, the values of density for the obtained composites increased from $0.28 \mathrm{~g} / \mathrm{cm}^{3}$ for pristine low-density BPR-derived carbon modified 3D needled carbon fiber composite to $0.59 \mathrm{~g} / \mathrm{cm}^{3}$ for S1 sample, to $0.77 \mathrm{~g} / \mathrm{cm}^{3}$ for S2 sample and further to $0.88 \mathrm{~g} / \mathrm{cm}^{3}$ for S3 sample, respectively. In addition, the dependence between density and PIP cycle number can be used to determine the microstructure and mechanical properties of the as-prepared composites.

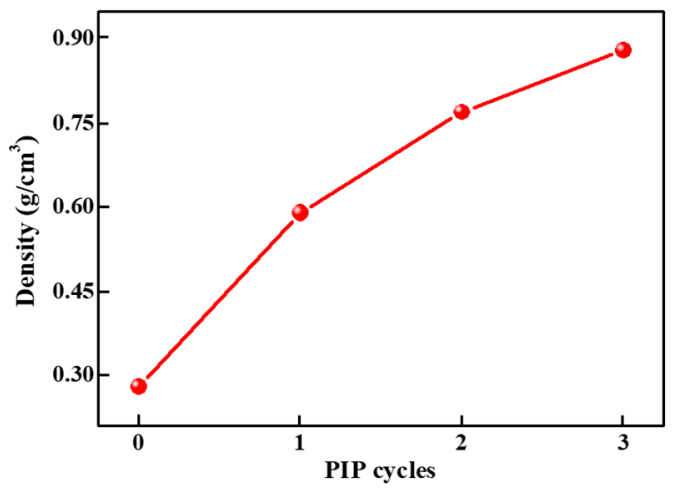

Figure 4. The densities of as-prepared composites with different pyrolysis process (PIP) cycle numbers. 
Secondly, the influence of PIP cycle numbers on the microstructure of as-prepared composites was observed by the SEM images listed in Figure 5. From the results, there the number of open pores within the composites decreases with increasing PIP cycle number, leading to an increase in density. Large open pores can be found in the composites after one PIP cycle. In contrast to this, only small voids could be observed after two PIP cycles. Then, the content of small voids decreases when the PIP cycle number increases to 3, and some local regions exhibit relatively dense. In addition, the presence of pores and voids would be beneficial to decrease the thermal conductivity of composites and improve the efficiency of thermal insulation, and further to tailor the impedance matching of composites.

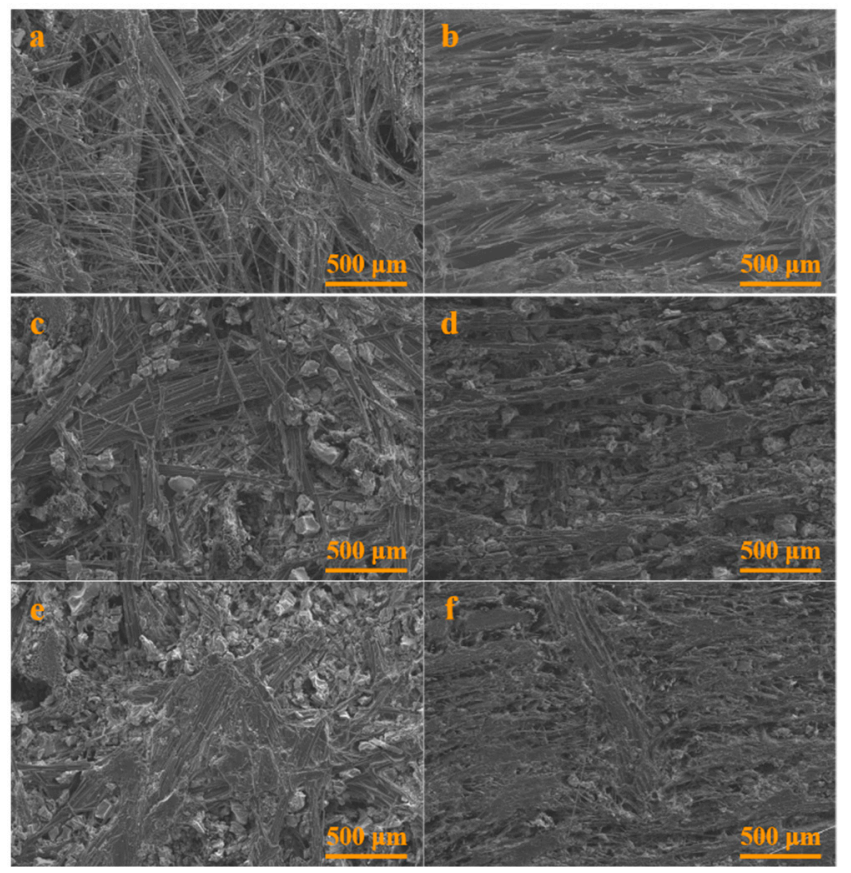

Figure 5. SEM images of as-prepared composites with different PIP cycle numbers from $X Y$ and $Z$ directions: $(\mathbf{a}, \mathbf{b})$ one PIP cycle; $(\mathbf{c}, \mathbf{d})$ two PIP cycles; $(\mathbf{e}, \mathbf{f})$ three PIP cycles.

Moreover, the effect of PIP cycle numbers on the compressive strength of as-prepared composites was investigated. Notably, the compressive strength of low-density composites with high porosities attracts more attention than that of tensile strength from the point of view of practical application, especially in the thermal protection system field, and thus the compressive strengths of as-prepared composites were evaluated and are displayed in Figure 6 [4]. The pristine compressive strength low-density BPR-derived carbon modified 3D needled carbon fiber composites without SiOC ceramic was just about $3.96 \pm 0.24 \mathrm{MPa}$ in $\mathrm{XY}$ direction and $1.86 \pm 0.13 \mathrm{MPa}$ in $\mathrm{Z}$ direction. From the results revealed in Figure 6, incorporation of SiOC ceramic would strongly improve the compressive strength of the composites, and the value of compressive strength increases both in the $X Y$ and $Z$ directions as the number of PIP cycle increases. Specifically, the compressive strengths for as-achieved samples with different PIP cycle numbers are about: $12.94 \pm 1.52 \mathrm{MPa}$ in XY direction and $6.65 \pm 0.98 \mathrm{MPa}$ in $Z$ direction for $S 1$ sample, $14.48 \pm 1.68 \mathrm{MPa}$ in $X Y$ direction and $8.62 \pm 1.03 \mathrm{MPa}$ in $Z$ direction for S2 sample, and $17.18 \pm 1.92 \mathrm{MPa}$ in $X Y$ direction and $9.01 \pm 1.25 \mathrm{MPa}$ in $Z$ direction for S3 sample, respectively. The reason for the different values of compressive strength between $X Y$ and $Z$ directions could be attributed to the distribution anisotropy of 3D needled carbon fiber preform, among which the most fiber orientation was parallel and perpendicular to $X Y$ and $Z$ directions, resulting in a much higher value at $X Y$ direction compared with that of $Z$ direction. 


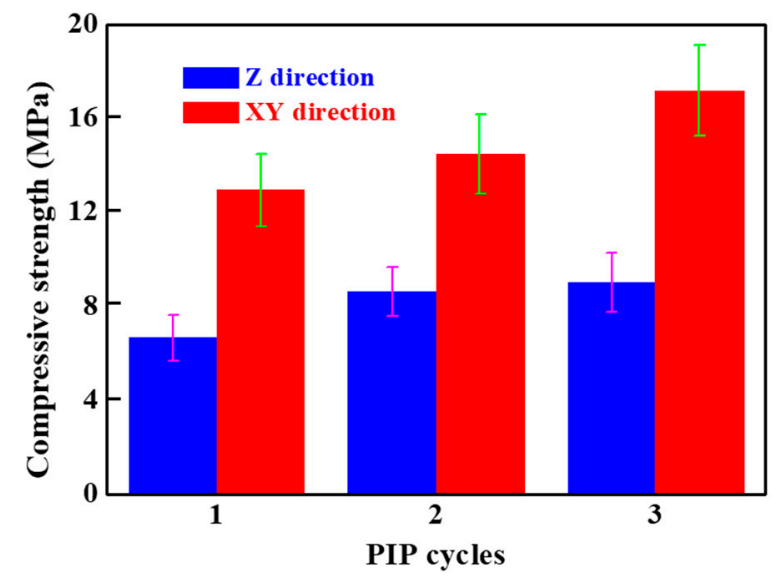

Figure 6. Compressive strengths of as-prepared composites with different PIP cycle numbers ranging from 1 to 3 times.

Besides the mechanical properties, high-temperature oxidation resistance and thermal stability of the materials are also important for the practical application. The high-temperature oxidation resistance and thermal stability properties of as-prepared composites were evaluated by TGA curves from room temperature to $1000^{\circ} \mathrm{C}$ both under air and Ar atmospheres, and the results of S3 sample and pristine low-density BPR-derived carbon modified 3D needled carbon fiber composite without $\mathrm{SiOC}$ ceramic are given in Figure 7. The results demonstrate that the introduction of SiOC would remarkedly improve the high-temperature oxidation resistance and thermal stability properties of low-density BPR-derived carbon modified 3D needled carbon fiber composites both under air and Ar atmospheres. It is worth pointing out that the weight reduction at low temperatures for the tested samples should be attributed to the absorbed moisture on the surface of the composites due to the high porosity. Delightedly, the S3 sample with SiOC ceramic presents excellent high-temperature oxidation resistance and thermal stability properties with weight retention above $95 \%$ up to about $1000{ }^{\circ} \mathrm{C}$ either under air or Ar atmosphere, indicating that the BPR-derived carbon modified 3D needled carbon fiber reinforced SiOC composites could be as a promising candidate of thermal protection materials for application in harsh environment. However, the low-density BPR-derived carbon modified 3D needled carbon fiber composite without SiOC ceramic exhibits poor high-temperature oxidation resistance and thermal stability properties, especially in air. Based on the above analysis results, it is reasonable to believe that the BPR-derived carbon modified 3D needled carbon fiber reinforced SiOC composites constructed and prepared in current work via a simple PIP method might be considered as a promising material employed in a harsh environment on account of their enhanced mechanical properties and excellent thermostabilities.
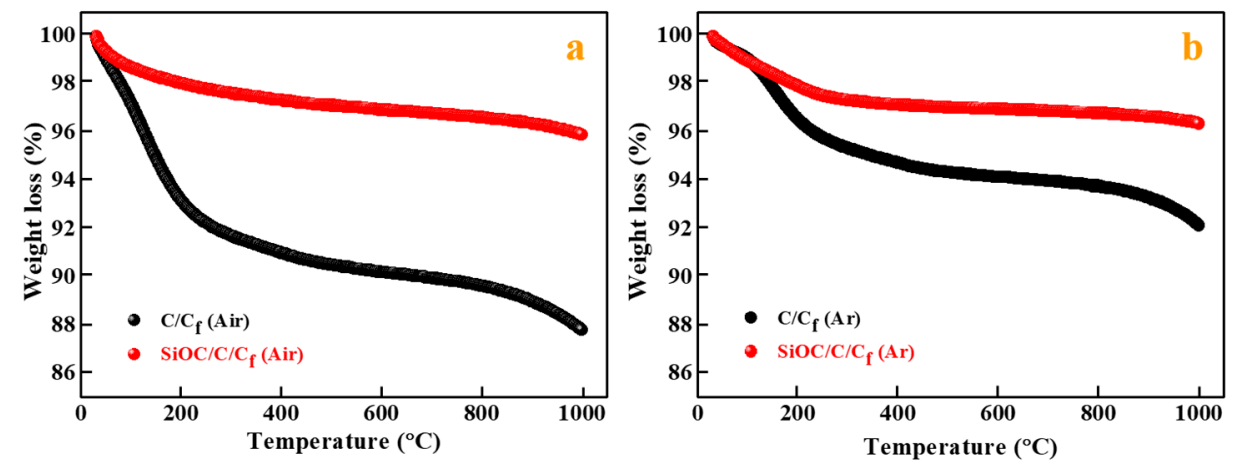

Figure 7. TGA curves for the as-prepared composites with three PIP cycles and composites without silicon oxycarbide (SiOC) ceramic from room temperature to $1000{ }^{\circ} \mathrm{C}$ under (a) air and (b) Ar atmospheres. 
Furthermore, the functional performances of BPR-derived carbon modified 3D needled carbon fiber reinforced SiOC composites, namely EMW absorption properties, were evaluated to open up the potential application fields. According to previous literature, the EMW absorption property of an absorber is closely related to its electromagnetic parameters, namely complex permittivity $\left(\varepsilon_{\mathrm{r}}=\varepsilon^{\prime}\right.$ $\left.-j \varepsilon^{\prime \prime}\right)$ and complex permeability $\left(\mu_{r}=\mu^{\prime}-j \mu^{\prime \prime}\right)$, while the value of $\mu_{r}$ could be equal to 1 due to not having a ferromagnetic phase involved [22,36]. Noteworthily, the real and imaginary parts of complex permittivity, namely $\varepsilon^{\prime}$ and $\varepsilon^{\prime \prime}$ relate to the storage and consume abilities of electric energy, and thus the relative complex permittivity of as-prepared composites with different PIP cycle numbers are given in Figure 8 [22,35]. From Figure 8a, it is clear that the $\varepsilon^{\prime}$ values for the composites increase first and then decrease as the PIP cycle number increases, whose value is connected with the polarization relaxation, while the $\varepsilon^{\prime}$ values of all samples decrease with increasing frequency apart from some slight rising. The decline tendency could be explained as following: the random dipoles within the sample would overcome the great resistance and exhibit preferential orientation paralleling to the external EM field, leading to the formation of dipole relaxation polarization, while there is not enough time for the rearrangement of the dipoles to respond to the rapidly changing external EM field, resulting in a declining tendency with the increase of frequency [29,37]. Similarly, the $\varepsilon^{\prime \prime}$ values for the composites shown in Figure $8 \mathrm{~b}$ also increase first and then decrease with increasing PIP cycle number, whose value strongly depends on the electrical conductivity of the tested sample, and thus the variation tendency for $\varepsilon^{\prime \prime}$ value should be attributed to the different content SiOC introduced after different PIP cycle numbers, which could be confirmed by the above result of density for different composites (in Figure 5). In addition, some peaks around at 4, 12, and $14 \mathrm{GHz}$ should be ascribed to the resonance behaviors [38,39]. Besides the above real and imaginary parts of complex permittivity, another critical electromagnetic parameter for an absorber is the dielectric tangent loss $\left(\tan \delta_{e}=\varepsilon^{\prime \prime} \mid \varepsilon^{\prime}\right)$, which presents the microwave attenuation capability, and thus the value of $\tan \delta_{e}$ was calculated and is displayed in Figure 8c. Likewise, the value of $\tan \delta_{e}$ increases first and then decreases with increasing PIP cycle number. The sequence for the average value of $\tan \delta_{e}$ for as-prepared composites is $\mathrm{S} 3<\mathrm{S} 2$ $<\mathrm{S} 1$, and a higher $\tan \delta_{e}$ value for an absorber without magnetic medium might own a better EMW absorption property from a theoretical point of view. As for the polarization, there are four polarizations easily created within a heterostructure, including electronic, atomic, Debye (dipolar), and interfacial polarizations [40]. According to previous results, the electronic and atomic polarizations would only be formed at a higher frequency, which could be excluded in the present study. As for interfacial polarization, it can be formed in the interface of two different components, and thus the interfacial polarizations would be formed at SiOC ceramic/BPR-derived carbon, SiOC ceramic/carbon fibers, and BPR-derived carbon/carbon fibers to improve the EMW absorption performances of the composites. Finally, as for the Debye polarization, which could be analyzed by the following the Cole-Cole curves. Based on the Debye theory, the relationship between $\varepsilon^{\prime}$ and $\varepsilon^{\prime \prime}$ could be written as follows $[29,38,41]$ :

$$
\left(\varepsilon^{\prime}-\frac{\varepsilon_{s}+\varepsilon_{\infty}}{2}\right)^{2}+\left(\varepsilon^{\prime \prime}\right)^{2}=\left(\frac{\varepsilon_{s}-\varepsilon_{\infty}}{2}\right)^{2}
$$

Notably, the parameters listed in the above equation, including $\varepsilon_{S}$ and $\varepsilon_{\infty}$ refers to the static permittivity and relative dielectric constant at the high-frequency limit. From Equation (5), the relationship between $\varepsilon^{\prime}$ and $\varepsilon^{\prime \prime}$ should be a semicircle, namely the Cole-Cole semicircle. The number of the Cole-Cole semicircle corresponds to the number of Debye relaxation processes. The Cole-Cole curve of all as-obtained samples with different PIP cycle numbers at the frequency $2-18 \mathrm{GHz}$ is displayed in Figure 8d. Clearly, at least four semicircles could be found among all the samples, indicating that various relaxation processes would be generated within all as-prepared composites. 

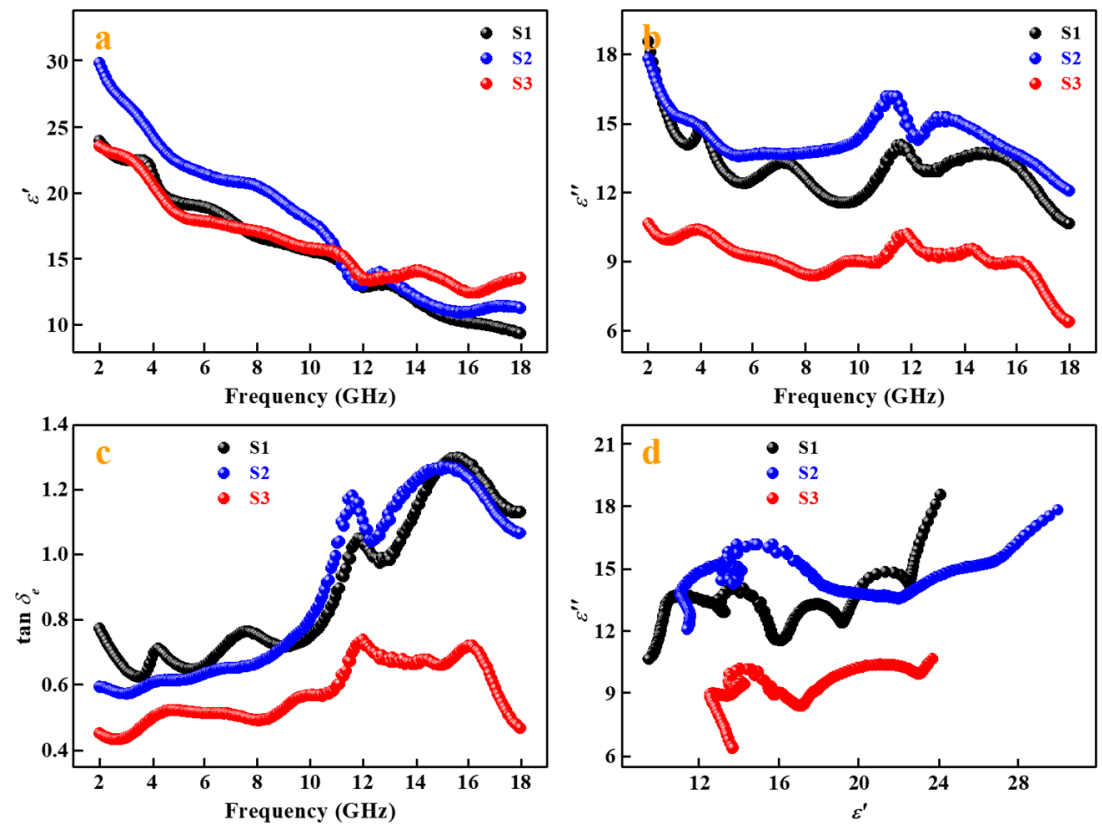

Figure 8. Frequency dependence of (a) the real part $\left(\varepsilon^{\prime}\right)$ and $(\mathbf{b})$ imaginary part $\left(\varepsilon^{\prime \prime}\right)$ of complex permittivity, and (c) dielectric loss $(\tan \delta e)$ and (d) Cole-Cole curves for as-prepared composites with different PIP cycle numbers in the frequency range of $2-18 \mathrm{GHz}$.

The EMW absorption properties of all samples with different PIP cycle numbers were evaluated, and the two-dimensional (2D) RL contour plots are revealed in Figure 9. The pristine low-density BPR-derived carbon modified 3D needled carbon fiber composite without SiOC ceramic possesses a poor EMW absorption performance just with an $R L_{\min }$ value of $-7 \mathrm{~dB}$, and the BPR-derived carbon modified 3D needled carbon fiber reinforced SiOC composites achieve substantially enhanced microwave absorption performances. From Figure $9 \mathrm{a}$, the $\mathrm{RL}_{\min }$ value for the $\mathrm{S} 1$ sample with one PIP cycle is about $-12.22 \mathrm{~dB}$ at $18 \mathrm{GHz}$ with a thin thickness of $1.00 \mathrm{~mm}$, and the EAB value is about 2.1 GHz (15.9-18.0 GHz). However, after increasing the PIP cycle number to 2, the $\mathrm{RL}_{\min }$ value is just about $-9.81 \mathrm{~dB}$ at the frequency of $18.00 \mathrm{GHz}$ and the matching thickness of $1.00 \mathrm{~mm}$. Obviously, the composite with three PIP cycle numbers (S3 sample) exhibits the best EMW absorption property, whose $\mathrm{RL}_{\min }$ value is as low as $-24.31 \mathrm{~dB}$ at $18 \mathrm{GHz}$ with an ultra-thin thickness of $1.05 \mathrm{~mm}$ (Figure 9c), and the largest EAB value for the $\mathrm{S} 3$ sample is about $4.9 \mathrm{GHz}$ ranging from $13.08 \mathrm{GHz}$ to $17.98 \mathrm{GHz}$ with the thickness of only $1.21 \mathrm{~mm}$. Combined with the above results, it is reasonable to believe that the introduction of SiOC ceramic would be beneficial for the enhancement of the EMW absorption properties of low-density BPR-derived carbon modified 3D needled carbon fiber composites, and their EMW absorption performances could be effectively controlled by just adjusting the PIP cycle numbers.
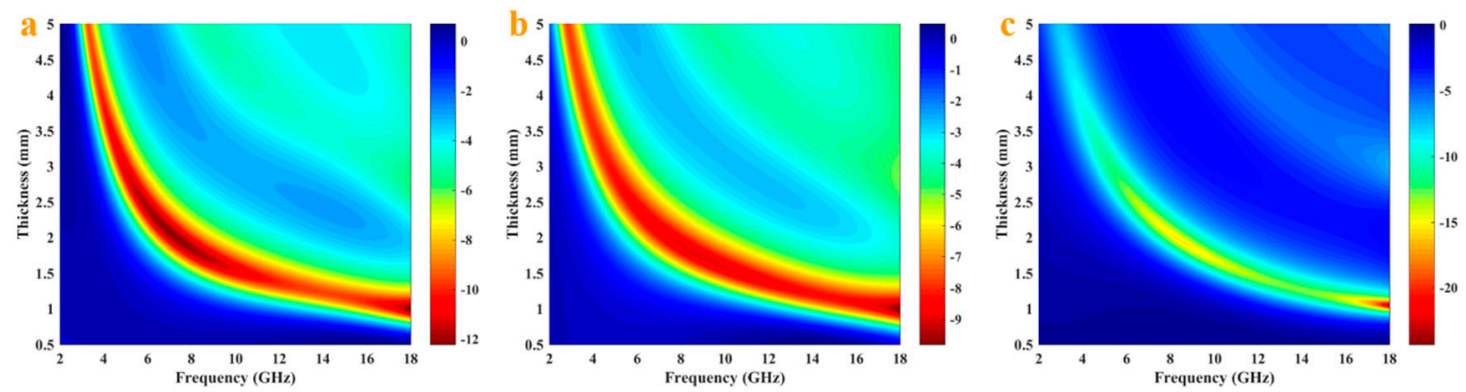

Figure 9. The 2D calculated reflection loss (RL) value maps of as-prepared composites with different PIP cycle numbers within $0.5-5 \mathrm{~mm}$ and 2-18 GHz: (a-c) one, two, and three PIP cycles. 
Reasons for the improved EMW absorption performance are described in the following: Firstly, the dipole and interfacial polarizations would be mainly responsible for the improved properties due to the presence of numerous Cole-Cole semicircles and interfaces [42,43]. Secondly, conductive and dielectric losses also tightly affected the EMW absorption performance because of the different conductivities among carbon fibers, BPR-derived carbon, and SiOC ceramic [42,43]. Moreover, the defects within the composites might also play an important role in increasing the microwave absorption abilities [42,43]. Overall, the enhanced EMW absorption properties might be mainly due to the matched impedance and improved loss ability that arose from the synergetic effect among carbon fibers, BPR-derived carbon, and SiOC ceramic. To date, lots of efforts have been made to investigate and improve the EMW absorption properties of carbon fibers and PDCs, especially in construction of composites, and thus we further compared the EMW absorption capabilities between our products and absorbers recently reported and the results were displayed in Figure 10 and Table S2 [6,9-13,15-20,24-26]. Although the $\mathrm{RL}_{\min }$ value for our products, namely BPR-derived carbon modified 3D needled carbon fiber reinforced SiOC composites is not the lowest, the value is comparable to those of most of PDCs (Figure 10a). Notably, our BPR-derived carbon modified 3D needled carbon fiber reinforced $\mathrm{SiOC}$ composites possess the widest $\mathrm{EAB}$ value among all the absorbers listed in the references, which is vitally important for design and construction of next-generation EMW absorption absorbers. The above results indicate that our BPR-derived carbon modified 3D needled carbon fiber reinforced SiOC composites with good high-temperature oxidation resistance, mechanical, and EMW absorption properties could be considered as a promising material for practical applications.
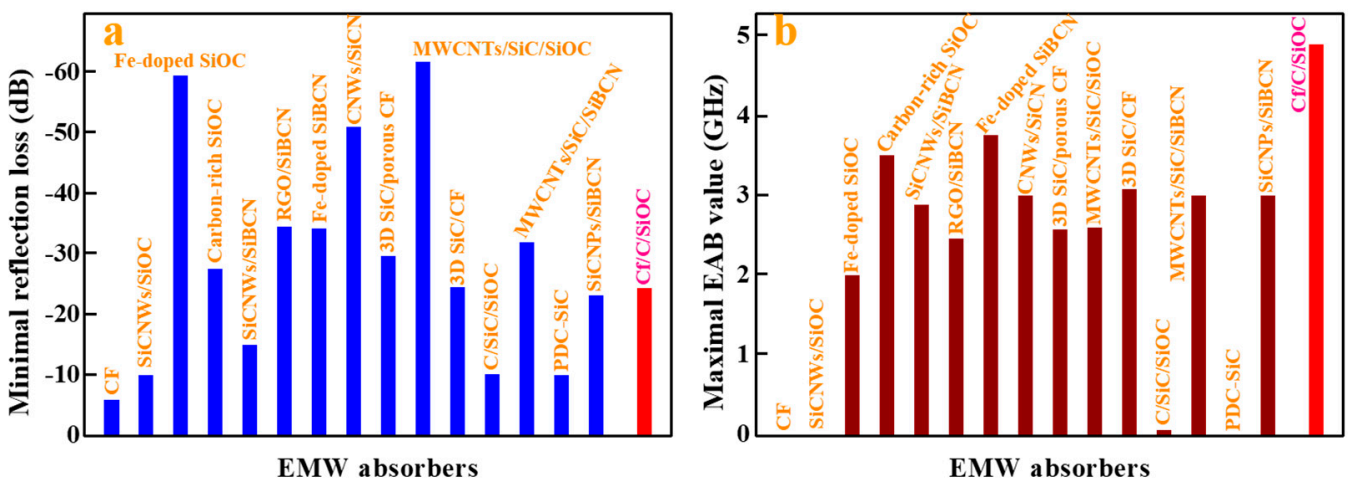

Figure 10. Comparison of (a) minimal reflection losses and (b) widest effective absorption bandwidth $(\mathrm{EAB})$ values of different electromagnetic wave (EMW) absorbers reported by recent literature.

\section{Conclusions}

In this paper, a novel composite composed of BPR-derived carbon modified 3D needled carbon fiber reinforced SiOC is presented and was constructed through a simple PIP method. The critical process parameter, namely the pyrolysis temperature was optimized. The influence of PIP cycle numbers on the microstructure, compressive strength, high-temperature oxidation resistance, and EMW absorption performances of the composites was studied for the first time, providing useful theory and experimental data for the research of PR-based composites.

The results show that the composition and microstructure of as-prepared products strongly rely on the pyrolysis temperature, as the $\mathrm{SiOC}$ phase will decompose to $\mathrm{SiC}, \mathrm{SiO}_{2}$, and free carbon when the temperature exceeds $1400^{\circ} \mathrm{C}$. In addition, the number of PIP cycles dramatically affects the microstructure and physical-mechanical properties of the composites. The density and compressive strength of the composites increases as the PIP cycle number is increased. In addition, the high-temperature oxidation resistance and thermal stability properties of the composites were remarkedly improved after the introduction of $\mathrm{SiOC}$, whose weight loss under both air and $\mathrm{Ar}$ atmosphere up to $1000{ }^{\circ} \mathrm{C}$ was less than $5 \%$. A substantial enhancement of EMW absorption properties was achieved after incorporation of SiOC ceramic compared with the poor microwave absorption 
ability of pristine low-density BPR-derived carbon modified 3D needled carbon fiber composite $\left(R L_{\min }=-7 \mathrm{~dB}\right)$, whose value of $R L_{\min }$ decreased to $-12.22 \mathrm{~dB}$ for the sample with one PIP cycle, to $-9.81 \mathrm{~dB}$ for ample with two PIP cycles and to $-24.31 \mathrm{~dB}$ for the sample with three PIP cycles.

In conclusion, the present study indicates that BPR-derived carbon modified 3D needled carbon fiber reinforced SiOC composites with good high-temperature oxidation resistance, mechanical, and EMW absorption properties could be considered as a potential candidate material for practical applications in a harsh environment. Our next work will focus on the optimize the overall performances of BPR-derived carbon modified 3D needled carbon fiber reinforced SiOC composites, especially in improving the high-temperature oxidation resistance with advanced multi-composition coating.

Supplementary Materials: The following are available online at http://www.mdpi.com/2076-3417/10/6/1924/s1.

Author Contributions: Y.S. (Yu Sun) performed the experiments and wrote the paper, and Y.S. (Yuguo Sun) designed the experiments and revised the paper. All authors have read and agreed to the published version of the manuscript.

Funding: This research received no external funding.

Acknowledgments: We thank all researchers for assistance in measurement and test samples.

Conflicts of Interest: The authors declare no conflict of interest.

\section{References}

1. Natali, M.; Kenny, J.M.; Torre, L. Science and technology of polymeric ablative materials for thermal protection systems and propulsion devices: A review. Prog. Mater. Sci. 2016, 84, 192-275. [CrossRef]

2. Yin, R.; Cheng, H.; Hong, C.; Zhang, X. Synthesis and characterization of novel phenolic resin/silicone hybrid aerogel composites with enhanced thermal, mechanical and ablative properties. Compos. Part A-Appl. S. 2017, 101, 500-510. [CrossRef]

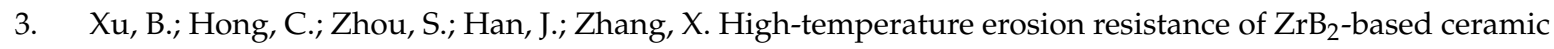
coating for lightweight carbon/carbon composites under simulated atmospheric re-entry conditions by high frequency plasma wind tunnel test. Ceram. Int. 2016, 42, 9511-9518. [CrossRef]

4. Sun, Y.; Hong, C.; Zhang, X.; Han, J.; Qu, Q. Preparation and properties of SiOC ceramic modified carbon fiber needled felt preform composites. Ceram. Int. 2020, 46, 1743-1749. [CrossRef]

5. Ma, Q.S.; Chen, Z.H.; Zheng, W.W.; Hu, H.F. Effects of pyrolysis processes on microstructure and mechanical properties of $\mathrm{Cf} / \mathrm{SiOC}$ composites fabricated by preceramic polymer pyrolysis. Ceram. Int. 2005, 31, 305-314. [CrossRef]

6. Yan, L.; Hong, C.; Sun, B.; Zhao, G.; Cheng, Y.; Dong, S.; Zhang, X. In situ growth of core-sheath heterostructural $\mathrm{SiC}$ nanowire arrays on carbon fibers and enhanced electromagnetic wave absorption performance. ACS Appl. Mater. Inter. 2017, 9, 6320-6331. [CrossRef]

7. Han, W.; Zhao, G.; Zhang, X.; Zhou, S.; Wang, P.; An, Y.; Xu, B. Graphene oxide grafted carbon fiber reinforced siliconborocarbonitride ceramics with enhanced thermal stability. Carbon 2015, 95, 157-165. [CrossRef]

8. Deng, P.; Shi, Y.; Liu, Y.; Liu, Y.; Wang, Q. Solidifying process and flame retardancy of epoxy resin cured with boron-containing phenolic resin. Appl. Surf. Sci. 2018, 427, 894-904. [CrossRef]

9. Song, C.; Cheng, L.; Liu, Y.; Zhao, M.; Ye, F. Microstructure and electromagnetic wave absorption properties of RGO-SiBCN composites via PDC technology. Ceram. Int. 2018, 44, 18759-18769. [CrossRef]

10. Duan, W.; Yin, X.; Li, Q.; Liu, X.; Cheng, L.; Zhang, L. Synthesis and microwave absorption properties of SiC nanowires reinforced SiOC ceramic. J. Eur. Ceram. Soc. 2014, 34, 257-266. [CrossRef]

11. Du, B.; He, C.; Shui, A.; Zhang, X.; Hong, C. Microwave-absorption properties of heterostructural SiC nanowires/SiOC ceramic derived from polysiloxane. Ceram. Int. 2019, 45, 1208-1214. [CrossRef]

12. Du, B.; Qian, J.; Hu, P.; He, C.; Cai, M.; Wang, X.; Shui, A. Enhanced electromagnetic wave absorption of Fe-doped silicon oxycarbide nanocomposites. J. Am. Ceram. Soc. 2020, 103, 1732-1743. [CrossRef]

13. Du, B.; He, C.; Qian, J.; Cai, M.; Wang, X.; Shui, A. Electromagnetic wave absorbing properties of glucose-derived carbon-rich SiOC ceramics annealed at different temperatures. J. Am. Ceram. Soc. 2019, 102, 7015-7025. [CrossRef] 
14. Niu, M.; Wang, H.; Su, L.; Zhang, D.; Shi, J. Fabrication and properties of lightweight SiOC modified carbon-bonded carbon fiber composites. Ceram. Int. 2016, 42, 10614-10618. [CrossRef]

15. Qin, H.; Liu, Y.; Ye, F.; Cheng, Z.; Chen, C.; Cheng, L.; Zhang, L. Dielectric and microwave absorption properties of SiCnw-SiBCN composite ceramics deposited via chemical vapor infiltration. J. Alloy. Compd. 2019, 771, 747-754. [CrossRef]

16. Li, W.; Li, X.; Gong, W.; Chen, P.; Zhu, Y.; Zhu, B. Construction of multiple heterogeneous interface and its effect on microwave absorption of SiBCN ceramics. Ceram. Int. 2019. [CrossRef]

17. Ren, F.; Yin, X.; Mo, R.; Ye, F.; Zhang, L.; Cheng, L. Hierarchical carbon nanowires network modified PDCs-SiCN with improved microwave absorption performance. Ceram. Int. 2019, 45, 14238-14248. [CrossRef]

18. Zhang, Y.; Yin, X.; Ye, F.; Kong, L. Effects of multi-walled carbon nanotubes on the crystallization behavior of PDCs-SiBCN and their improved dielectric and EM absorbing properties. J. Eur. Ceram. Soc. 2014, 34, 1053-1061. [CrossRef]

19. Li, Q.; Yin, X.; Duan, W.; Kong, L.; Hao, B.; Ye, F. Electrical, dielectric and microwave-absorption properties of polymer derived $\mathrm{SiC}$ ceramics in X band. J. Alloy. Compd. 2013, 565, 66-72. [CrossRef]

20. Ye, F.; Zhang, L.; Yin, X.; Zhang, Y.; Kong, L.; Liu, Y.; Cheng, L. Dielectric and microwave-absorption properties of $\mathrm{SiC}$ nanoparticle/SiBCN composite ceramics. J. Eur. Ceram. Soc. 2014, 34, 205-215. [CrossRef]

21. Hong, W.; Dong, S.; Hu, P.; Luo, X.; Du, S. In situ growth of one-dimensional nanowires on porous PDC-SiC/Si $3 \mathrm{~N}_{4}$ ceramics with excellent microwave absorption properties. Ceram. Int. 2017, 43, 14301-14308. [CrossRef]

22. Duan, W.; Yin, X.; Ye, F.; Li, Q.; Han, M.; Liu, X.; Cai, Y. Synthesis and EMW absorbing properties of nano SiC modified PDC-SiOC. J. Mater. Chem. C 2016, 4, 5962-5969. [CrossRef]

23. Luo, C.; Tang, Y.; Jiao, T.; Kong, J. High-temperature stable and metal-free electromagnetic wave-absorbing $\mathrm{SiBCN}$ ceramics derived from carbon-rich hyperbranched polyborosilazanes. ACS Appl. Mater. Inter. 2018, 10, 28051-28061. [CrossRef] [PubMed]

24. Ye, X.; Chen, Z.; Ai, S.; Hou, B.; Zhang, J.; Liang, X.; Cui, S. Effects of SiC coating on microwave absorption of novel three-dimensional reticulated $\mathrm{SiC}$ /porous carbon foam. Ceram. Int. 2019, 45, 8660-8668. [CrossRef]

25. Chen, L.; Zhao, J.; Wang, L.; Peng, F.; Liu, H.; Zhang, J.; Guo, Z. In-situ pyrolyzed polymethylsilsesquioxane multi-walled carbon nanotubes derived ceramic nanocomposites for electromagnetic wave absorption. Ceram. Int. 2019, 45, 11756-11764. [CrossRef]

26. Ye, X.; Chen, Z.; Ai, S.; Hou, B.; Zhang, J.; Liang, X.; Cui, S. Porous SiC/melamine-derived carbon foam frameworks with excellent electromagnetic wave absorbing capacity. J. Adv. Ceram. 2019, 8, 479-488. [CrossRef]

27. Liu, C.; Meng, X.; Zhang, X.; Hong, C.; Han, J.; Han, W.; Du, S. High temperature structure evolution of macroporous SiOC ceramics prepared by a sol-gel method. Ceram. Int. 2015, 41, 11091-11096. [CrossRef]

28. Dong, S.; Hu, P.; Zhang, X.; Han, J.; Zhang, Y.; Luo, X. Carbon foams modified with in-situ formation of $\mathrm{Si}_{3} \mathrm{~N}_{4}$ and $\mathrm{SiC}$ for enhanced electromagnetic microwave absorption property and thermostability. Ceram. Int. 2018, 44, 7141-7150. [CrossRef]

29. Dong, S.; Zhang, W.; Zhang, X.; Hu, P.; Han, J. Designable synthesis of core-shell SiCw@ C heterostructures with thickness-dependent electromagnetic wave absorption between the whole X-band and Ku-band. Chem. Eng. J. 2018, 354, 767-776. [CrossRef]

30. Dong, S.; Song, J.; Zhang, X.; Hu, P.; Sun, B.; Zhou, S.; Luo, X. Strong contribution of in situ grown nanowires to enhance the thermostabilities and microwave absorption properties of porous graphene foams under different atmospheres. J. Mater. Chem. C 2017, 5, 11837-11846. [CrossRef]

31. Pena-Alonso, R.; Mariotto, G.; Gervais, C.; Babonneau, F.; Soraru, G.D. New insights on the high-temperature nanostructure evolution of SiOC and B-doped SiBOC polymer-derived glasses. Chem. Mater. 2007, 19, 5694-5702. [CrossRef]

32. Gao, Y.; Li, L.; Tan, P.; Liu, L.; Zhang, Z. Application of Raman spectroscopy in carbon nanotube-based polymer composites. Chin. Sci. Bull. 2010, 55, 3978-3988. [CrossRef]

33. Sasaki, K.I.; Wakabayashi, K.; Enoki, T. Polarization dependence of Raman spectra in strained graphene. Phys. Rev. B 2010, 82, 205407. [CrossRef] 
34. Hu, P.; Dong, S.; Zhang, X.; Gui, K.; Chen, G.; Hu, Z. Synthesis and characterization of ultralong SiC nanowires with unique optical properties, excellent thermal stability and flexible nanomechanical properties. Sci. Rep.-UK 2017, 7, 1-10. [CrossRef]

35. Dong, S.; Zhang, X.; Zhang, D.; Sun, B.; Yan, L.; Luo, X. Strong effect of atmosphere on the microstructure and microwave absorption properties of porous SiC ceramics. J. Eur. Ceram. Soc. 2018, 38, 29-39. [CrossRef]

36. Bi, S.; Su, X.; Hou, G.; Liu, C.; Song, W.L.; Cao, M.S. Electrical conductivity and microwave absorption of shortened multi-walled carbon nanotube/alumina ceramic composites. Ceram. Int. 2013, 39, 5979-5983. [CrossRef]

37. Song, W.L.; Cao, M.S.; Hou, Z.L.; Yuan, J.; Fang, X.Y. High-temperature microwave absorption and evolutionary behavior of multiwalled carbon nanotube nanocomposite. Scripta Mater. 2009, 61, 201-204. [CrossRef]

38. Dong, S.; Lyu, Y.; Li, X.; Chen, J.; Zhang, X.; Han, J.; Hu, P. Construction of MnO nanoparticles anchored on $\mathrm{SiC}$ whiskers for superior electromagnetic wave absorption. J. Colloid Interf. Sci. 2020, 559, 186-196. [CrossRef]

39. Xie, S.; Guo, X.N.; Jin, G.Q.; Guo, X.Y. Carbon coated Co-SiC nanocomposite with high-performance microwave absorption. Phys. Chem. Chem. Phys. 2013, 15, 16104-16110. [CrossRef]

40. Seïd, K.A.; Badot, J.C.; Dubrunfaut, O.; Levasseur, S.; Guyomard, D.; Lestriez, B. Multiscale electronic transport mechanism and true conductivities in amorphous carbon- $\mathrm{LiFePO}_{4}$ nanocomposites. J. Mater. Chem. 2012, 22, 2641-2649. [CrossRef]

41. Ding, D.; Wang, Y.; Li, X.; Qiang, R.; Xu, P.; Chu, W.; Du, Y. Rational design of core-shell Co@C microspheres for high-performance microwave absorption. Carbon 2017, 111, 722-732. [CrossRef]

42. Qiao, M.; Lei, X.; Ma, Y.; Tian, L.; He, X.; Su, K.; Zhang, Q. Application of yolk-shell Fe $\mathrm{O}_{4} @$ N-doped carbon nanochains as highly effective microwave-absorption material. Nano Res. 2018, 11, 1500-1519. [CrossRef]

43. Wang, P.; Cheng, L.; Zhang, Y.; Wu, H.; Hou, Y.; Yuan, W.; Zheng, L. Flexible, hydrophobic SiC ceramic nanofibers used as high frequency electromagnetic wave absorbers. Ceram. Int. 2017, 43, 7424-7435. [CrossRef] 